\title{
Slowing Down of Neutrons in Infinite Homogeneous Media $\uparrow$
}

\author{
BY N. CORNGOLD \\ Brookhaven National Laboratory, Upton, New York \\ MS. received 7th Fune 1957, and in final form 30th $\mathscr{7 u l y} 1957$
}

4bstract. We have derived from the Boltzmann equation a new integral equation governing the slowing down of neutrons in a homogeneous mixture of atoms. The new equation reflects a view of the neutrons as propagating down the energy scale, whereas the Boltzmann equation expresses a collision balance. Unlike the Boltzmann equation the new equation lends itself to approximate solution by teration and to the construction of an accurate variational principle for $p$, the esonance escape probability. Some numerical results are given.

\section{\$1. INTRODUCTION}

$\Gamma$ HE nature of the slowing down of fast neutrons produced uniformly throughout homogeneous media composed of atoms of arbitrary mass was discussed ten years ago in a definitive paper by Placzek (1946). Placzek's paper was most successful in dealing with media which did not absorb neutrons. In a later review paper, Marshak (1947), using Laplace transform techniques, treated large variety of slowing-down problems but in analysing absorbing media he dealt principally with absorption cross sections which varied slowly with energy. The very important case of absorption in resonances has not received adequate :eatment in the literature.

In unpublished notes E. P. Wigner and A. M. Weinberg have recently shown that the Boltzmann equation for slowing down in an infinite media composed of sne type of atom is equivalent to another, new, integral equation. The new iquation is then used to obtain a correction to the usual expression for the capture mobability in slowing down in such a media. The argument given by Wigner ind Weinberg in deriving the new equation is essentially 'physical'. It is our purpose in this paper to show: (i) that the transformation to the new equation ray be easily effected by the use of the Laplace transformation, (ii) that the Laplace transformation method enables one to construct the new equation for biving down in arbitrary mixtures of atoms, a generalization that is not trivially bious from the 'physical' argument, and (iii) that a variational principle, based in the equation, may be given for the capture probability. Examples that are : lien show that the variational method is accurate and may be of considerable ractical importance.

We begin our development with rederivation of the Wigner-Weinberg quation. In fact, much of this work was completed before the author was aware the unpublished work.

tWork performed under the auspices of the U.S. Atomic Energy Commission.

PROC. PHYS. SOC. LXX, II-A 


\section{§ 2. Mathematical Development}

The traditional approach to the slowing-down problem in homogeneous media of infinite extent is based upon the Boltzmann equation for the collision density

$$
\Phi(u)=\int_{0}^{\prime \prime} d u^{\prime} K_{m}\left(u-u^{\prime}\right) h\left(u^{\prime}\right) \Phi\left(u^{\prime}\right)+S(u) .
$$

Here $u$, the logarithmic energy, is related to neutron energy by $u=\ln \left(E_{0} \mid E\right)$, $\Phi(u) d u$ is the number of collisions taking place per unit time in the interval between $u$ and $u+d u$ per unit volume of matter, and $S(u) d u$ is the number of source neutrons introduced into unit volume in unit time with energies between $u$ and $u+d u$. The scattering medium is composed of atoms of mass $m$. The kernel $K_{m}\left(u-u^{\prime}\right)$ characterizes the scattering of neutrons by the atoms and is found to be

$$
\begin{aligned}
K_{m}\left(u-u^{\prime}\right) & =\frac{\exp \left\{-\left(u-u^{\prime}\right)\right\}}{1-\alpha_{m}} & & \text { when } q_{m}>u-u^{\prime}>0 \\
& =0 & & \text { elsewhere }
\end{aligned}
$$

where $q_{m}=\ln [(m+1) /(m-1)]^{2}$ is the maximum logarithmic energy loss for a neutron upon collision and $1-\alpha_{m}=4 m /(1+m)^{2}$. Finally, $h(u)$ is the probablity that a neutron-nucleus collision at energy $u$ will be a scattering collision. In (1) $h(u)$ may be alternatively written as the ratio of scattering to total cross sections at energy $u$. For a detailed discussion of the derivation of (1) the reader may consult Marshak's review.

Equation (1) expresses a balance of collisions; the left-hand side represents collisions throwing the neutron out of energy $u$, the integral term in the right hand side represents collisions of faster neutrons, scattered into $u$. Except for the cases of very light scatterers (hydrogen, deuterium, ...) the integrand is zero except for energies quite close to $u$. That is, $q_{m} \ll 1$. In cases of practical interest, the source is at a fixed energy $E_{0}$, whence $S(u)=S \delta(u)$ and one is most concerned with neutrons that have made many collisions in the moderating material $\left(u \gg q_{m}, u>1\right)$. Equation (1) because of its 'local' character is poorly fitted to give information about $\Phi(u)$ in this energy region, when the source has been specified. Iteration upon the source function, or numerical integration requires that one follow the neutrons through their many collisions from source energy to $u$, a procedure that generally requires the assistance of a computing machine.

In the special case $h(u) \equiv 1$, when there is no possibility for absorption, equation (1) may be solved. The solution, which we designate by $\psi_{m}(u)$ approaches, for large $u$, the constant value $1 / \xi_{m} . \quad \xi_{m}$ is the average logarithmic energy loss per collision and is given by

$$
\xi_{m}=1-\frac{q_{m} \exp \left(-q_{m}\right)}{1-\alpha_{m}}=1+\frac{(m-1)^{2}}{2 m} \ln \left(\frac{m-1}{m+1}\right) .
$$

For smaller $u\left(u \sim q_{m}\right) \psi_{m}(u)$ varies rapidly and shows, in this domain, discontinuttes in function and derivatives. We have assumed here, as we shall in what follows, that $S(u)=\delta(u)$.

We begin the derivation of the new equation by separating from $\Phi(u)$ " singular component composed of neutrons at source energy that have not yet made a first collision. We put $\Phi(u)=\phi(u)+\delta(u)$ and upon substitution of this expression into equation (1), obtain

$$
\phi(u)=\int_{0}^{u} d u^{\prime} K_{m}\left(u-u^{\prime}\right) h\left(u^{\prime}\right) \phi\left(u^{\prime}\right)+h(0) K_{m}(u) .
$$


Vext, we apply a Laplace transformation to equation (5). If we denote the transformation

$$
\int_{0}^{\infty} d u \mathrm{e}^{-\eta n} f(u) \text { by } \mathrm{L}\{f(u)\}=\tilde{f(\eta)}
$$

and remember the result of the convolution theorem

$$
\mathrm{L}\left\{\int_{0}^{u} d u^{\prime} f\left(u-u^{\prime}\right) g\left(u^{\prime}\right)\right\}=\tilde{f}(\eta) \tilde{g}(\eta),
$$

equation (5) becomes

$$
\tilde{\phi}(\eta)=\widetilde{K}_{m}(\eta) L\{h \phi\}+h(0) \widetilde{K}_{m}(\eta)
$$

If we now write $h(u)=1-g(u)$, where $g(u)$ is the capture probability, equation (8) becomes

$$
\tilde{\phi}(\eta)=\frac{h(0) \widetilde{K}_{m}(\eta)}{1-K_{m}(\eta)}-\frac{\widetilde{K}_{m}(\eta)}{1-\widetilde{K}_{m}(\eta)} \mathbf{L}(g \phi)
$$

low we recognize that $\widetilde{K}_{m}(\eta) /\left(1-\tilde{K}_{m}(\eta)\right)$ is the transform of the solution $\psi_{m}$ to the no-capture equation, whereupon inversion of (9) yields the new equation

$$
\phi(u)=h(0) \psi_{m}(u)-\int_{0}^{u} d u^{\prime} \psi_{m}\left(u-u^{\prime}\right) g\left(u^{\prime}\right) \phi\left(u^{\prime}\right)
$$

The physical content of the equation shows up if we transfer the integral term to the lett-hand side. We then observe that $\phi(u)$, the solution in the case of capture is, when corrected by the integral term, equal to the solution of the no-capture case. The correction consists in adding neutrons to compensate for absotptions; e.g. the number of neutrons removed per unit time between $u^{\prime}$ and $u^{\prime}+d u^{\prime}$ is $\left\{u^{\prime} u^{\prime} \phi\left(u^{\prime}\right) d u^{\prime}\right.$. A source of the same strength will produce a 'disturbance' $f_{i}^{\prime}\left(u^{\prime} \phi\left(u^{\prime}\right) d u^{\prime} \psi_{m}\left(u-u^{\prime}\right)\right.$ at energy $u$. Thus, the picture is reminiscent of the multiple scattering of waves. Neutron density introduced at source energy 'propagates' according to $\psi_{m}(u)$. Variations in cross section disturb the propagaton at $u_{1}, u_{2}, \ldots$ producing secondary waves which propagate according to $b_{m}\left(u-u_{1}\right), \psi_{m}\left(u-u_{2}\right)$, etc. The secondary waves interfere with the primary Tave to produce the resultant, $\phi$.

The mathematical operations leading from the Boltzmann equation (1). to the 'new' equation (10) may also be applied in the case of an $N$-component, homogeneous mixture of atoms. The Boltzmann equation is

$$
\Phi(u)=\sum_{s=1}^{N} \int_{0}^{u} d u^{\prime} K m_{s}\left(u-u^{\prime}\right) h_{s}\left(u^{\prime}\right) \Phi\left(u^{\prime}\right)+\delta(u)
$$

where the $K m_{s}$ are scattering kernels as described in equation (2). $h_{s}(u)$ has a somewhat different meaning. It is now the probability that a collision at energy $u$. nill be a scattering collision with an atom of type $s$. Thus

$$
h_{s}(u)=n_{s} \sigma_{\mathrm{scatt}}(\mathrm{s}) / \sum_{r=1}^{N} n_{r} \sigma_{\text {tot }}{ }^{(r)}
$$

and when absorption is absent, $h_{s}$ is not equal to unity. In equation (12) $n_{r}$ is the number of atoms of type $r$ per unit volume.

Proceeding as in the previous case, we obtain for the transformed equation:

$$
\tilde{\phi}(\eta)=\frac{\sum_{s} h_{s}(0) \widetilde{K}_{s}(\eta)}{1-\sum_{s} h_{s}(0) \widetilde{K}_{s}(\eta)}-\frac{\sum_{s} \widetilde{K}_{s}(\eta) L\left\{g_{s} \phi\right\}}{1-\sum_{s} h_{s}(0) \widetilde{K}_{s}(\eta)}
$$


with $g_{s}(u)$ defined by $h_{s}(0)-h_{s}(u)=g_{s}(u)$. In the case of a mixture, then, $g_{8}$ is not proportional to the absorption by a type-s nucleus. $g_{s}$ vanishes only if the absorption vanishes and the scattering is independent of energy.

If we define the function $\bar{\psi}_{s}$ by :

$$
\mathrm{L}\left\{\bar{\psi}_{s}\right\}=\tilde{K}_{s}(\eta) /\left[1-\sum_{r} h_{r}(0) \tilde{K}_{r}(\eta)\right]
$$

or, equivalently

$$
\bar{\psi}_{s}(u)=\sum_{r=1}^{N} h_{r}(0) \int_{0}^{u} d u^{\prime} K_{r}\left(u-u^{\prime}\right) \bar{\psi}_{s}+K_{s}(u)
$$

inversion of (13) yields:

$$
\phi(u)=\psi(u)-\sum_{s} \int_{0}^{u} d u^{\prime} \bar{\psi}_{s}\left(u-u^{\prime}\right) g_{s}\left(u^{\prime}\right) \phi\left(u^{\prime}\right)
$$

with $\psi(u)=\Sigma_{s} h_{s}(0) \bar{\psi}_{s}(u)$.

In $(16), \psi(u)$ is the solution to the slowing-down problem in the mixture, provided that the cross sections are non-absorptive, and energy independent. $\bar{\psi}_{s}$ is another solution in the same non-absorptive mixture, but corresponds to a different initial condition. $\bar{\psi}_{s}$ describes the slowing down of neutrons whose first collision is with a nucleus of type $s$. Clearly, $\psi(u)=\Sigma_{s} h_{s}(0) \bar{\psi}_{s}(u)$. For sufficiently large $u$ the $\bar{\psi}_{s}$ become identical with each other and with $\psi(u)$.

Equation (16) may be pictured in a manner similar to the discussion of equation (10). Neutrons propagate energy-wise from source energy according to the function $\psi(u)$. When absorption $\left(g_{s}>0\right)$ or ' anomalous' scattering $\left(g_{s}<0\right)$ occurs, the distribution is perturbed. A perturbation caused by collision with a types nucleus may be compensated by the appropriate source $g_{s}\left(u^{\prime}\right) \phi\left(u^{\prime}\right)$. Neutrons propagating from this source do so according to $\bar{\psi}_{s}\left(u-u^{\prime}\right)$ since their first collsion is with a type-s nucleus. The integral in (16) sums the effect of the compensating sources-the scattered waves.

\section{\$3. Solving the New Equations}

We are not able to give exact general solutions of (10) and (16), just as we are unable to solve equation (1) exactly. However, unlike equation (1), good approxlmate solutions to the new equations may be constructed by iteration. For equation (10) iteration yields a series solution: $(h(0)=1)$

$$
\begin{aligned}
\phi(u)=\psi_{m}(u) & -\int_{0}^{u} d u^{\prime} \psi_{m}\left(u-u^{\prime}\right) g\left(u^{\prime}\right) \psi_{m}\left(u^{\prime}\right) \\
& +\int_{0}^{u} d u^{\prime} \psi_{m}\left(u-u^{\prime}\right) g\left(u^{\prime}\right) \int_{0}^{u^{\prime}} d u^{\prime \prime} \psi_{m}\left(u^{\prime}-u^{\prime \prime}\right) g\left(u^{\prime \prime}\right) \psi_{m}\left(u^{\prime \prime}\right) \\
& -\ldots,
\end{aligned}
$$

which is composed of successively higher powers of absorption probability and will converge rapidly in all practical cases. The overall probability for capture of a neutron which is slowing down is seldom greater than $40 \%$. 
If, in equation (17) the functions $\psi_{m}$ are replaced by their asymptotic values $\lim \psi_{m}(u)=1 / \xi_{m}$, the series may be summed to give $\lim _{x \rightarrow \infty}$

$$
\phi(u)=\frac{1}{\xi_{m}} \exp \left\{-\int_{0}^{u} g\left(u^{\prime}\right) d u^{\prime}\right\} .
$$

This expression is approximate, and well known. It is an exact solution of (17) only when the function $g(u)$ has resonances of vanishingly small width separated by $\Delta u \gg q_{m}$.

A quantity of considerable interest is the probability, $p$, that a neutron will escape capture during the slowing-down process. $g_{s}(u)$, which governs capture, differs from zero only in a small range of $u$ (and energy), the 'resonance region'. $\downarrow$ simple way to obtain $p$ is to compare the asymptotic (large $u$ ) collision density obtained when capture is present with asymptotic density in the no-capture case. The ratio of densities is $p$. If we examine (16) in the limit $u \rightarrow \infty$, noting that $g(u)$ ranishes after we pass through the resonance region, we find the asymptotic values of $\phi$ and $\psi$ related by

$$
\phi_{\mathrm{as}}=\psi_{\mathrm{as}}-\lim _{u \rightarrow \infty} \sum_{\delta} \int_{0}^{u} d u^{\prime} \bar{\psi}_{s}\left(u-u^{\prime}\right) g_{s}\left(u^{\prime}\right) \phi\left(u^{\prime}\right) .
$$

$\bar{\psi}_{s}$ as we have noted, describes slowing-down in a mixture. Whether a neutron begins its life with a collision with a type $s$ or a type $r$ or $\cdot$ nucleus in the mixture itsasymptotic behaviour will be the same. Thus $\lim _{u \rightarrow \infty} \bar{\psi}_{s}(u)=\bar{\psi}_{a s}$ independent of $s$. Since $\psi(u)=\sum_{s} h_{s}(0) \bar{\psi}_{s}(u)$ and $\sum_{s} h_{s}(0)=1$ in a medium characterized by nonabsorptive, energy-independent cross sections,

$$
\bar{\psi}_{\mathrm{as}} \equiv \lim _{u \rightarrow \infty} \bar{\psi}_{s}(u)=\psi_{\mathrm{as}}=\lim _{u \rightarrow \infty} \sum_{\delta} h_{s}(0) \bar{\psi}_{s}(u)
$$

The asymptotic value $\psi_{\mathrm{as}}$ is well known: it is $\psi_{\mathrm{as}}=1 / \xi_{\mathrm{av}}, \xi_{\mathrm{av}}=\Sigma_{s} h_{s}(0) \xi_{s}$. Hence

$$
\phi_{\mathrm{as}}=\frac{1}{\xi_{\mathrm{av}}}\left[1-\int_{0}^{\infty} d u^{\prime} \sum_{s} g_{s}\left(u^{\prime}\right) \phi\left(u^{\prime}\right)\right]=\frac{1}{\xi_{\mathrm{av}}} p .
$$

Using now (20) and (16) it is possible to construct the expression $J\left[\phi, \phi^{+}\right]=$

$$
\frac{\int_{0}^{\infty} d u \sum_{s} g_{s}(u) \phi^{+}(u) \phi(u)+\int_{0}^{\infty} d u \sum_{s} g_{s}(u) \phi^{+}(u) \int_{0}^{u} d u^{\prime} \sum_{l} \bar{\psi}_{l}\left(u-u^{\prime}\right) g_{l}\left(u^{\prime}\right) \phi\left(u^{\prime}\right)}{\int_{0}^{\infty} d u^{\prime} \sum_{s} g_{s}\left(u^{\prime}\right) \phi\left(u^{\prime}\right) \int_{0}^{\infty} d u^{\prime} \sum_{s} g_{s}\left(u^{\prime}\right) \phi^{+}\left(u^{\prime}\right)}
$$

with the following properties: if $\phi(u)$ is a solution to $(16), J\left[\phi, \phi^{+}\right]=1 /\left[\xi_{\text {av }}(1-p)\right]$. If $\phi(u)$ deviates by $\delta \phi(u)$ from the solution to $(16)$ and $\phi^{+}(u)$ deviates by $\delta \phi^{+}(u)$ from the solution to an appropriate adjoint equation, the change in $J[\cdot \cdot], \delta J$, vanishes to second order in $\delta \phi$ and $\delta \phi^{+}$. Thus $J[\cdot \cdot]$ is stationary with regard to changes in $\phi$ and $\phi^{+}$, and the right-hand side of (21) should give an accurate value for $p$ when crude guesses for $\phi$ and $\phi^{+}$are substituted therein. We have constructed a variational principle for $p$.

While the proof of the stationary character of (21) will be relegated to the Appendix, we should mention here that the assumption has been made that for values of $u$ in the resonance region, where $g_{s}(u)$ is non-zero, $\psi(u)$ has reached its 
asymptotic value, $1 /\left[\xi_{\text {av }}\right]$. If we do not wish to make this very plausible assump. tion, the correct $J\left[\phi, \phi^{+}\right]$differs from (21) in that the factor

$$
\int_{0}^{\infty} d u^{\prime} \sum_{s} g_{s}\left(u^{\prime}\right) \phi^{+}\left(u^{\prime}\right) \text { is replaced by } \int_{0}^{\infty} d u^{\prime} \sum_{s} g_{s}\left(u^{\prime}\right) \phi^{+}\left(u^{\prime}\right) \psi\left(u^{\prime}\right)
$$

and for the correct $\phi, \phi^{+}, J\left[\phi, \phi^{+}\right]=1 /(1-p)$. The stationary character is unaltered. With the assumption mentioned above, it is easy to show that

$\phi(u)=\frac{1}{\xi_{\mathrm{av}}} \frac{1}{1-p} \int_{0}^{\infty} d u^{\prime} \sum_{s} g_{s}\left(u^{\prime}\right) \phi\left(u^{\prime}\right)-\int_{0}^{u} d u^{\prime} \sum_{s} \vec{\psi}_{s}\left(u-u^{\prime}\right) g_{s}\left(u^{\prime}\right) \phi\left(u^{\prime}\right)$

while the discussion in the Appendix shows that $\phi^{+}(u)$ must satisfy

$$
\begin{aligned}
\phi^{+}(u)=\frac{1}{\xi_{\text {av }}} & \frac{1}{1-p} \int_{0}^{\infty} d u^{\prime} \sum_{s} g_{s}\left(u^{\prime}\right) \phi^{+}\left(u^{\prime}\right) \\
& -\int_{u}^{\infty} d u^{\prime} \sum_{s} g_{s}(u) \bar{\psi}_{s}\left(u^{\prime}-u\right) \frac{\sum_{l} g_{l}\left(u^{\prime}\right)}{\sum_{l} g_{l}(u)} \phi^{+}\left(u^{\prime}\right) .
\end{aligned}
$$

If we specialize to a one-component mixture, (22) and (23) become

$$
\begin{aligned}
\phi(u) & =\lambda \int_{0}^{\infty} d u^{\prime} g\left(u^{\prime}\right) \phi\left(u^{\prime}\right)-\int_{0}^{u} d u^{\prime} \psi_{m}\left(u-u^{\prime}\right) g\left(u^{\prime}\right) \phi\left(u^{\prime}\right) \\
\phi^{+}(u) & =\lambda \int_{0}^{\infty} d u^{\prime} g\left(u^{\prime}\right) \phi^{+}\left(u^{\prime}\right)-\int_{u}^{\infty} d u^{\prime} \psi_{m}\left(u^{\prime}-u\right) g\left(u^{\prime}\right) \phi^{+}\left(u^{\prime}\right)
\end{aligned}
$$

with $\lambda=1 /[\xi(1-p)]$.

\section{§4. Applications of the Variational Principle}

A variation-iteration scheme, based on equations (21) and (22-23) or (24) will enable the investigator to obtain $p$ to any desired degree of accuracy, in a systematic manner. In this scheme a zero-order set of trial functions is used to get $J\left[\phi_{0}, \phi_{0}{ }^{+}\right]$and $p_{0}$. Then $\phi_{0}, \phi_{0}{ }^{+}$and $p_{0}$ are substituted into the right-hand sides of equations (22-23) or (24) in order to give $\phi_{1}, \phi_{1}{ }^{+} . J\left[\phi_{1}, \phi_{1}{ }^{+}\right]$then yields $p_{1}$ and the process continues. One might suppose the procedure to involve a large amount of computational effort. We shall show, however, that considerable accuracy is obtained-in some simple examples-with only the zero-order and first-order trial functions.

\subsection{A Mixture of Hydrogen and Infinitely Heavy Absorber}

The solution to this problem is well known. For hydrogen, $\psi_{m}=\psi_{1} \equiv 1$, and either iteration or differentiation of equation (16) leads to the solution

$$
p=\exp \left\{-\int_{0}^{\infty} d u g(u)\right\}=\mathrm{e}^{-4}
$$

Using $\phi_{0}=1, \phi_{0}{ }^{+}=1$ in equation (21) we find

$$
p_{0}=\left(1-\frac{1}{2} A\right) /\left(1+\frac{1}{2} A\right)=1-A+\frac{1}{2} A^{2}-\cdots
$$

which agrees with the exact $p$ up to terms in third order in the absorption. If we now iterate upon $\phi_{0}, \phi_{0}{ }^{+}$and $p_{0}$, to get $\phi_{1}$ and $\phi_{1}{ }^{+}$, we find

$$
p_{1}=\left(1-\frac{1}{2} A+\frac{1}{12} A^{2}\right) /\left(1+\frac{1}{2} A+\frac{1}{12} A^{2}\right),
$$

which agrees with the exact $p$ to terms in fifth order in the absorption. Indeed $p_{1}$ yields $5 / 6$ of the fifth order term. 


\subsection{A Mixture of Hydrogen and ${ }^{238} U$}

Here we shall compare the lowest order estimate of $p, p_{0}$, with exact numerical alculations for a mixture with $1: 1$ atom ratio of hydrogen to uranium, in which only a single absorption resonance of the uranium is considered. The numerical calculations, communicated to the author by Mr. K. T. Spinney, were based on a differential equation obtained from equation (11). We list also the results given by wo other well-known approximations, the 'infinite absorber' (IA) in which the moderating effects of uranium are neglected and formula (25) is used, and the 'narrow resonance' (NR) in which the collision densities $\bar{\psi}_{s}$ are replaced by their asymptotic values $1 / \xi_{\text {av }}$. The calculation of $J\left[\phi_{0}, \phi_{0}^{+}\right]$which involves the construction of $\psi_{0}$ and $\psi_{1}$ (see Appendix) requires numerical integration, but much lesslabour is expended than in the ' exact' numerical calculation.

$\begin{array}{ccccc}\text { Resonance } & 1-p \text { (exact) } & 1-p_{0} \text { (variational) } & 1-p(\mathrm{NR}) & 1-p(\text { IA) } \\ 36.9 \mathrm{ev} & 0.05820 & 0.05682 & 0.04739 & 0.06110 \\ 192 \mathrm{ev} & 0.007119 & 0.007630 & 0.005068 & 0.01228\end{array}$

\section{§ 5. SUMMARY}

We have derived from the Boltzmann equation a new integral equation governing the slowing down of neutrons in a homogeneous mixture of atoms. The new equation reflects a view of the neutrons as propagating down the energy scale, whereas the Boltzmann equation expresses a collision balance. The new equation lends itself to approximate solution by iteration and to the construction of an accurate variational principle for $p$, the resonance escape probability. Its greater tractability should help investigators in the study of slowing-down processes. In particular, interference effects in the capture by nearby levels will be elucidated.

\section{REFERENCES}

Marshak, R. E., 1947, Rev. Mod. Phys., 19, 185.

PlaczeK, G., 1946, Phys. Rev., 69, 423.

\section{A P PENDIX}

\section{The Variational Principle}

Wewish to prove that $J\left[\phi, \phi^{+}\right]$defined in equation (21) is stationary with respect to arbitrary, small variations of $\phi, \phi^{+}$from the solutions to equations $(22,23)$.

$$
\begin{aligned}
& J\left[\phi, \phi^{+}\right]\left\{\int_{0}^{\infty} d u \sum_{s} g_{s} \phi\right\}\left\{\int_{0}^{\infty} d u \sum_{s} g_{s} \phi^{+}\right\} \\
& =\int_{0}^{\infty} d u \sum_{s} g_{s} \phi \phi^{+}+\int_{0}^{\infty} d u \sum_{s} g_{s} \phi^{+}(u) \int_{0}^{u} d u^{\prime} \sum_{l} \bar{\psi}_{l} g_{l} \phi\left(u^{\prime}\right) .
\end{aligned}
$$
find

If we vary $\phi^{+}$by $\delta \phi^{+}$from the solution to equation (23) and collect terms we

$$
\begin{aligned}
& \delta J \int_{0}^{\infty} d u \sum_{s} g_{s} \phi^{+} \int_{0}^{\infty} d u \sum_{s} g_{s} \phi=\int_{0}^{\infty} d u \sum_{s} g_{s}(u) \delta \phi^{+}(u)[F] \\
& -F=J \int_{0}^{\infty} d u \sum_{s} g_{s} \phi-\int_{0}^{u} d u^{\prime} \sum \bar{\psi}_{l}\left(u-u^{\prime}\right) g_{l}\left(u^{\prime}\right) \phi\left(u^{\prime}\right)-\phi(u) .
\end{aligned}
$$


Since $\phi$ satisfies equation (22) and $J\left[\phi, \phi^{+}\right]=1 /\left[(1-p) \xi_{\text {av }}\right] F$ must be zero. Since the coefficients of $\delta J$ do not in general vanish, $\delta J=0$. If we instead vary $\phi$ by $\delta \phi$ from the solution to equation (22) we find

$$
\begin{gathered}
\delta J \int_{0}^{\infty} d u \sum_{s} g_{s} \phi^{+} \int_{0}^{\infty} d u \sum_{s} g_{s} \phi=\int_{0}^{\infty} d u \sum_{s} g_{s}(u) \delta \phi(u)[F] \\
F=\phi^{+}-J \int_{0}^{\infty} d u \sum_{\delta} g_{s} \phi^{+}+\int_{u}^{\infty} d u^{\prime} \sum_{\delta} g_{l}(u) \psi_{l}\left(u^{\prime}-u\right) \frac{\sum_{s} g_{s}\left(u^{\prime}\right)}{\sum_{s} g_{s}(u)} \phi^{+}\left(u^{\prime}\right) .
\end{gathered}
$$

Thus if $\phi$ satisfies equation (23) $J$ will again be zero. We have shown, then, the stationary character of $J\left[\phi, \phi^{+}\right]$, when $\phi, \phi^{+}$are the actual solutions to the integral equations.

\section{Construction of the $\bar{\psi}_{8}$ Functions}

We shall indicate how one would construct the functions $\bar{\psi}_{2}$ and $\bar{\psi}_{1}$ relating to the slowing down of neutrons in a two-component mixture, here composed of uranium (subscript 2, and hydrogen (subscript 1).

By definition

$$
\begin{aligned}
\bar{\psi}_{s} & =\frac{h_{1}(0)}{1-\alpha_{1}} \int_{\gamma_{1}\left(u-q_{1}\right)}^{u} d u^{\prime} \exp \left[-\left(u-u^{\prime}\right)\right] \bar{\psi}_{s}\left(u^{\prime}\right) \\
& +\frac{h_{2}(0)}{1-\alpha_{2}} \int_{\gamma_{2}\left(u-q_{2}\right)}^{u} d u^{\prime} \exp \left[-\left(u-u^{\prime}\right)\right] \bar{\psi}_{s}\left(u^{\prime}\right)+\frac{1-\gamma_{s}}{1-\alpha_{s}} \mathrm{e}^{-u^{\prime \prime}},
\end{aligned}
$$

where $\gamma_{s}=\gamma_{s}(u)=0$ for $u \leqslant q_{s}$

$$
=1 \quad u>q_{s} \text {. }
$$

If we write $h_{s}(0) /\left(1-\alpha_{s}\right)=c_{s}, \mathrm{e}^{u_{\psi_{s}}}=y_{s}$ and then go to our example of hydrogen + uranium, (31) becomes

$$
y_{s}=c_{1} \int_{0}^{u} d u^{\prime} y_{s}\left(u^{\prime}\right)+c_{2} \int_{\gamma_{2}\left(u-q_{2}\right)}^{u} d u^{\prime} y_{s}\left(u^{\prime}\right)+\frac{1-\gamma_{s}}{1-\alpha_{s}} .
$$

For

$$
u \leqslant q_{2} \quad y_{s}=\left(c_{1}+c_{2}\right) \int_{0}^{u} d u^{\prime} y_{s}\left(u^{\prime}\right)+\frac{1}{1-\alpha_{s}}
$$

and

$$
y_{s}=\frac{\exp \left[\left(c_{1}+c_{2}\right) u\right]}{1-\alpha_{s}} \text {. }
$$

For $u>q_{2} \quad y_{s}=\left(c_{1}+c_{2}\right) \int_{0}^{u} d u^{\prime} y_{s}\left(u^{\prime}\right)-c_{2} \int_{0}^{u-q_{2}} d u^{\prime} y_{s}\left(u^{\prime}\right)+\frac{1-\gamma_{s}}{1-\alpha_{s}}$.

Clearly, $y_{2}$ is discontinuous at $u=q_{2}$, whlle $y_{1}$ is continuous there. In addition, differentiation of (33) and (34) shows that the first derivative of $y_{2}$ is discontinuous at $u=q_{2}, 2 q_{2}$, the second derivative at $u=q_{2}, 2 q_{2}, 3 q_{2}$, etc. These results would be expected to follow at once from Placzek's discussion.

If we differentiate (34), re-arrange terms and, if $(n+1) q_{2} \geqslant u \geqslant n q_{2}$, integrate between $n q_{2}$ and $u$, we find

where

$$
x_{s}(u)=x_{s}\left(n q_{2}{ }^{+}\right)-c_{2} \exp \left\{-q_{2}\left(c_{1}+c_{2}\right)\right\} \int_{(n-1) q_{s}}^{u-q_{s}} x_{s}\left(u^{\prime}\right) d u^{\prime}
$$

and

$$
\begin{aligned}
& x_{s}(u)=\left(1-\alpha_{s}\right) \exp \left\{-\left(c_{1}+c_{2}\right) u\right\} y_{s}(u) \\
& x_{s}\left(n q_{2}{ }^{+}\right)=\lim _{\varepsilon \rightarrow 0} x_{s}\left(n q_{2}+\epsilon\right) \quad \epsilon>0 .
\end{aligned}
$$

The 'limit from above' is introduced in order properly to take discontinuites into account. Equation (35) is the fundamental equation for the construction of 
$j_{\text {. U }}$ Using the result $x_{8}=1$ for $u \leqslant q_{2}$ one can generate $x_{s}(u)$ for higher values of $u$ through successive simple integrations in (35). The integrations may be one analytically or numerically.

Both $\bar{\psi}_{1}$ and $\bar{\psi}_{2}$ should approach the asymptotic value $1 / \xi_{\text {av }}$ for large $u$. While tis always difficult to extract the precise value of the constant from the Boltzmann quation, we can verify that (35) has a solution $x_{s}(u)=\exp \beta_{s} u$ where $\beta_{s}$ is independent of $s$ and has the value $\beta_{s}=1-\left(c_{1}+c_{2}\right)$, whence $\bar{\psi}_{s}(u)=$ const.

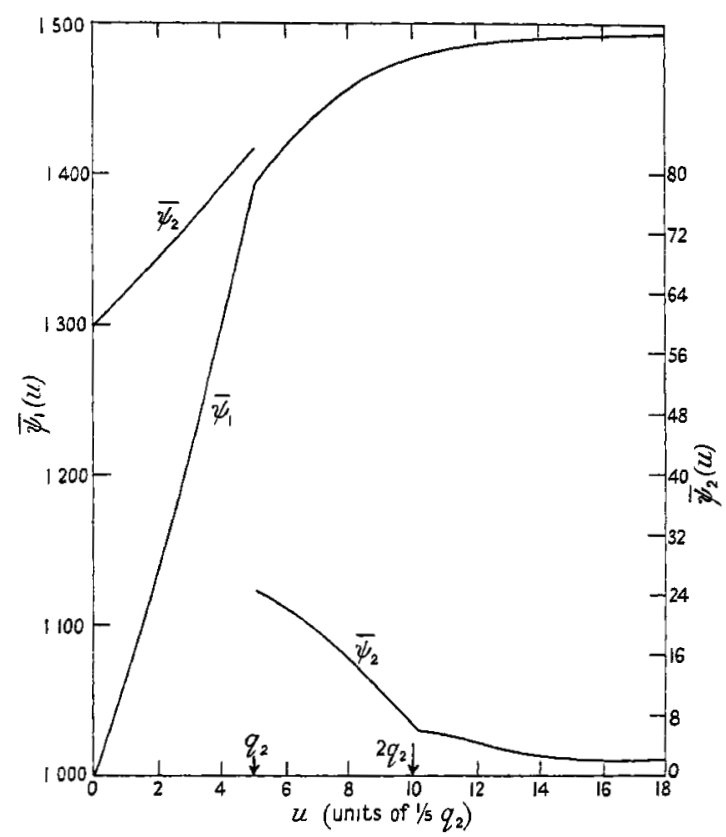

The functions $\bar{\psi}_{1}$ and $\bar{\psi}_{2}$ for $1: 1$ atom ratio of hydrogen to uranium.

The figure shows a graph of the functions $\bar{\psi}_{1}(u)$ and $\bar{\psi}_{2}(u)$ for the $1: 1$ atom ratio mentioned in the text. The functions $\bar{\psi}_{1}$ and $\bar{\psi}_{2}$ rapidly approach their asymptotic ialue. 